\title{
The Major Instrumentation Program at the National Science Foundation
}

\author{
G.X. Tessema ${ }^{1}$ \\ ${ }^{1}$ National Science Foundation, National Facilities and Instrumentation Program, Division of \\ Materials Research, Alexandria, USA. \\ The Major Research Instrumentation (MRI) program at the National Science Foundation (NSF) \\ is the main mechanism for funding major research instruments such as electron microscopy at \\ US universities and colleges. The MRI Program catalyzes new knowledge and discoveries by \\ empowering the Nation's scientists and engineers with state-of-the-art research instrumentation. \\ The Program enables research-intensive learning environments that promote the development of \\ a diverse workforce and next generation instrumentation, as well as facilitates academic/private \\ sector partnerships.
}

Among the goals of the MRI Program are: 1) Supporting the acquisition of major state-of-the-art instrumentation, thereby improving access to, and increased use of, modern research and research training instrumentation by a diverse workforce of scientists, engineers, and graduate and undergraduate students; 2) Fostering the development of the next generation of instrumentation, resulting in new instruments that are more widely used, and/or open up new areas of research and research training; 3) Enabling academic departments, disciplinary and cross-disciplinary units, and multi-organization collaborations to create well-equipped research environments that integrate research with research training; 4) Supporting the acquisition and development of instrumentation that contributes to, or takes advantage of, existing investments in cyberinfrastructure, while avoiding duplication of services already provisioned by NSF investments; 5) Promoting substantive and meaningful partnerships for instrument development between the academic and private sectors.

Such partnerships have the potential to build capacity for instrument development in academic settings and to create new products with wide scientific and commercial impact. The program receives about 800 proposals annually requesting funds for instrument development or acquisition.

This talk will describe recent changes in the MRI program and address questions about proposal preparation such as: What makes an MRI proposal fail before it is reviewed? What can make an MRI proposal actually succeed? What are the common mistakes to avoid? What should be the balance between focus on well-defined science drivers vs. breadth to serve a broad number of users?...and more. 


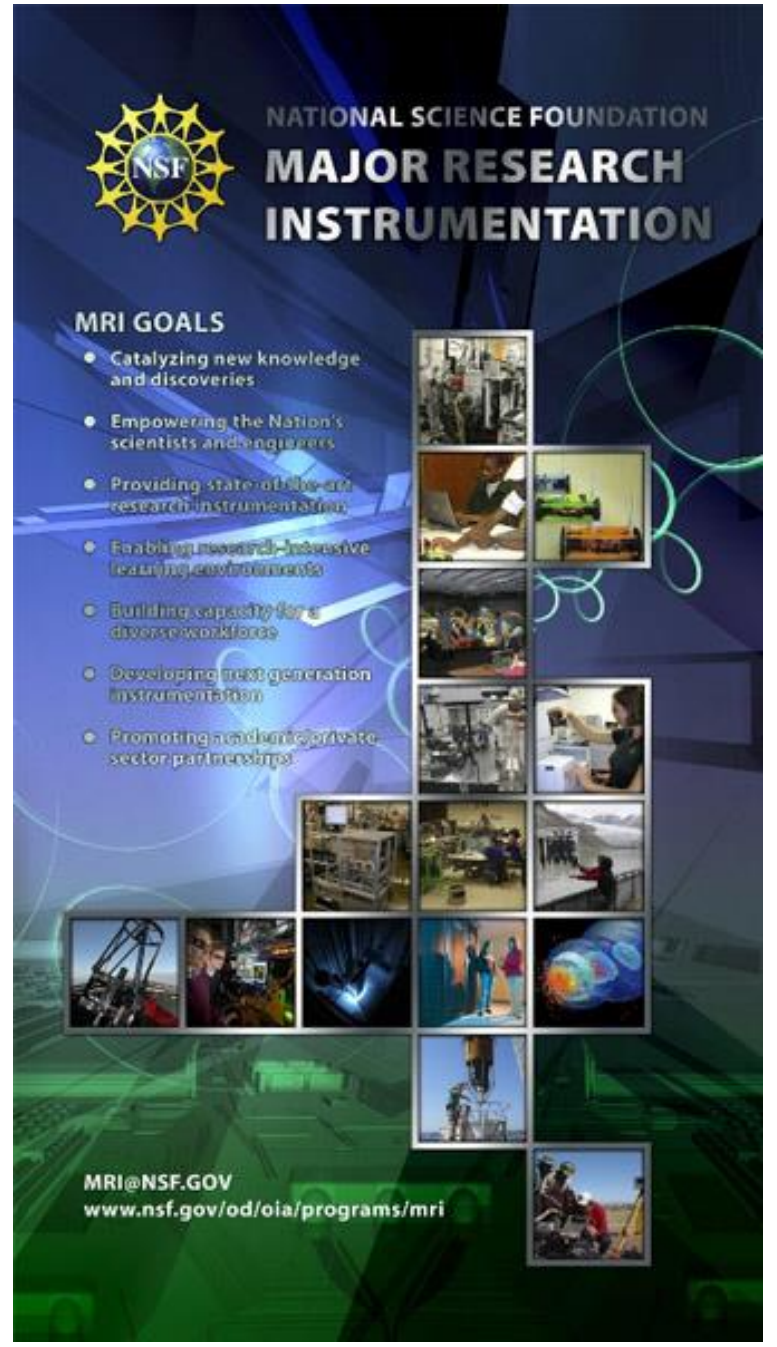

Figure 1. Splash page for the Major Research Instrumentation program of the National Scienc Foundation. 